\title{
Impact of Imperfect Information on the Optimal Exercise Strategy for Warrants
}

\author{
April 2008
}

\begin{abstract}
In this paper, we determine the optimal exercise strategy for corporate warrants if investors suffer from imperfect information and we evaluate the impact of this friction on the value of a warrant. For this purpose, we address both exercises at maturity, where imperfect information about the firm value is present, and exercises before maturity which are impacted by imperfect information about the size of the dividend. We model imperfect information so that all warrant holders know that they obtain biased signals of the true state without observing the signals of other warrant holders. The optimal exercise strategy follows from a complex game among warrant holders in which every individual warrant holder must account for the potential signals of the other warrant holders and their resulting exercise decisions. The main findings are that due to imperfect information warrant holders optimally start to exercise their warrants later than without imperfect information. Moreover, a simple block exercise strategy is always an equilibrium strategy for a high degree of imperfect information before maturity, even though a partial exercise can be the unique strategy without imperfect information. Remarkably, imperfect information does not necessarily result in a lower warrant value. As long as a warrant holder has a signal that allows for correct exercise decisions, then imperfect information enhances the warrant value due to suboptimal exercises by other investors.
\end{abstract}

JEL Classification: G13, C70

Keywords: Warrant Exercise; Block Exercise; Imperfect Information; Global Games 


\section{Introduction}

Corporate warrants are an important instrument especially for small and medium firms that want to issue (speculative) debt. Many of those firms attach warrants to their bonds in order to 'sweeten' a new issue (see Standard \& Poor's (2007)). As Noddings/Christoph/Noddings (1998) report, more than 10,000 firms in the US have warrants outstanding among which 55 percent have a remarkably low market capitalization below $\$ 100$ million.

As a consequence of the low firm size of many companies using warrants, the shares of those firms do not necessarily trade frequently. Therefore, at the relevant exercise dates of warrants there might not be any reliable observation of the share price. This is particularly true if the trading volume of the shares of the corresponding firm is very low, the last available market price of this share is several weeks (or even months) old, and the firm exhibits a relatively high volatility of the equity value return. In this case, the last price does not necessarily reflect the true value due to the low trading volume. In addition, the high volatility can result in a strong deviation between the true value at the last trading date and the true value at the current examination date. For this reason, different investors can have considerably different estimates of the true firm value at a given point in time. This fundamental implication introduces the problem that the standard assumption in the warrant pricing literature, namely that exercise decisions can be based on the current value of the firm, is often in conflict with the practical challenge to exercise a warrant. Rather investors face a problem concerning imperfect information, because they know that not every warrant holder has the same evaluation for the corresponding firm value. Therefore, it is crucial for the understanding of the optimal exercise strategy of warrant holders to know about the consequences from imperfect information among them.

Even in the standard case without imperfect information, the optimal warrant exercise decision, i.e. to buy a given number of new shares at a pre-specified strike price or to keep the warrants unexercised, is not straightforward. The optimal strategy rather results from a game among warrant holders (see e.g. Emanuel (1983), Constantinides (1984), and Spatt/Sterbenz (1988)). This is a consequence of the fact that a warrant exercise means that the proceeds from the payment of the strike price go to the firm and the number of shares outstanding increases. In other words, an exercise implies a higher firm value that is divided among more shares. Therefore, the exercise value a given warrant holder obtains does not only depend on his 
or her exercise decision but also on the decisions of all the other warrant holders. Emanuel (1983) and Constantinides (1984) argue that not only warrant exercise strategies with no or a complete exercise (block exercise) are optimal but also a partial exercise can be the optimal exercise strategy of this game in equilibrium. The seminal paper by Constantinides (1984), in which he analyzes optimal warrant exercises, was the basis for further examinations of the optimal exercise strategy. While Constantinides regards an especially simple model with perfect competition among the warrant holders and with a capital structure including only stocks and warrants, there are extensions in terms of the competition among warrant holders (see e.g. Cox/Rubinstein (1985), Spatt/Sterbenz (1988) and Shalem (2003)) and in terms of a more general capital structure including not only stocks and warrants but also additional debt (see Koziol (2006a) and Linder/Trautmann (2006)).

In this paper, we attribute importance to the fact that many warrant issuing firms are small so that different warrant holders can have different beliefs about the relevant parameters for the exercise decision. To evaluate the question whether imperfect information among investors has a relevant impact on the exercise decision or not, we first take a look at call options. In the case of a call option exercise (rather than a warrant exercise), the call option holder obtains an already existing share so that the total number of outstanding shares remains unaffected from call option exercises. In order to model imperfect information, we can have the notion that every investor gets a signal, e.g. for the true firm value, where depending on this signal the exercise decision must be made. If investors are risk neutral, they can base their investment decisions on the conditionally expected value of their wealth. It is straightforward to see that call option holders always exercise, if the signal they obtain is above a critical barrier. This critical barrier, however, does not depend on the existence of imperfect information because the conditionally expected values of a share and a warrant for a given signal are the same with and without imperfect information. This is because the exercise decision of the call option holders does not affect the prices of the underlying share so that strategic effects between option holders do not matter. Therefore, call option holders can use the same block exercise strategy as without imperfect information, where they exercise whenever the signal they obtain exceeds the critical barrier.

This simple argument for call options, that imperfect information does not affect the exercise decision, does not necessarily apply to warrants. This is a result of the fact that the exercise decisions of the other warrant holders are crucial for the exercise value every single warrant holder obtains. Thus, every warrant holder must account for the exercise decisions of the other warrant holders and its impact on 
the expected exercise value. For example, if most of the other warrant holders get a more favorable signal than a given warrant holder, the total exercise volume is possibly higher than in the case that most of the other investors obtain a worse signal. As a consequence, the presence of imperfect information complicates the determination of the optimal warrant exercise strategies and might therefore have an impact on the critical exercise barriers.

The goal of this paper is to determine the optimal exercise strategy for warrants under imperfect information and its impact on warrant values. With this analysis, we want to understand whether imperfect information affects the optimal exercise strategy or whether the same critical exercise barriers can be used as without imperfect information which is true for call options. Given that imperfect information has an effect on the optimal exercise strategy, it is desirable to derive a clear rule to adjust the optimal exercise, which can be applied by individual warrant holders if problems of imperfect information are present. For this analysis, we assume an especially simple model like in Constantinides (1984) with firms having only stocks and warrants outstanding and with perfect competition among the warrant holders to focus on the effects of imperfect information. We regard both optimal exercise strategies at maturity and before maturity. While at maturity problems of imperfect information about the firm value are present, we assume that before maturity imperfect information about the size of the total dividend is the major difficulty for the exercise decision. This is a relevant aspect because in real markets warrants must usually be exercised some months before the dividend date to participate in the dividend payment which can create imperfect information about the dividend. To model imperfect information, we regard a global games environment as proposed by Carlsson/van Damme (1993) which is closely related to the frameworks applied by Hubert/Schäfer (2002) and Morris/Shin (2004) that analyze coordination problems between a borrower and multiple lenders. Investors obtain different signals about the firm value (or the dividend) and know that there is imperfect information but they cannot observe how their signal is related to those of the other investors.

We find that imperfect information fundamentally affects the optimal exercise strategy of warrants. In general, exercises under imperfect information require more favorable signals for the firm value than without imperfect information. On the contrary, we observe that a complete exercise before maturity can occur even for less favorable dividend signals than without imperfect information. A further finding is that for a high degree of imperfect information a block strategy is an equilibrium before maturity, while without imperfect information a partial exercise is always the unique strategy for some dividends. Even though imperfect information com- 
plicates the exercise decision, the warrant value can benefit from this informational problem. Given that a warrant holder has a signal that allows (very likely) for a correct exercise decision, then imperfect information enhances the warrant value due to suboptimal exercises by other investors.

The paper is organized as follows: In Section 2, we describe the model for the analysis at maturity and discuss the optimal exercise strategies with and without imperfect information. Furthermore, we compare the resulting warrant values with and without informational problems. Section 3 contains the corresponding analysis for the optimal exercise strategy before maturity, where problems of imperfect information about the dividend are present. Section 4 concludes. A technical development is in Appendix A.

\section{Imperfect Information About the Firm Value}

\subsection{Model Framework}

We consider a firm that has issued $n$ shares and $m$ warrants that are still outstanding at the maturity date $T$ of the warrants. One warrant allows its holder to buy one newly issued share at the strike price $E$. If a fraction $f \in[0,1]$ of the $m$ outstanding warrants is exercised at maturity, the value of an exercised warrant $W_{T}\left(V_{T}, f \cdot m\right)$ for a given firm value $V_{T}$ results in:

$$
W_{T}\left(V_{T}, f \cdot m\right)=\frac{V_{T}+f \cdot m \cdot E}{n+f \cdot m}-E=\frac{V_{T}-n \cdot E}{n+f \cdot m} .
$$

As a consequence of the exercise, the firm value $V_{T}$ before exercise increases due to the exercise proceeds to $V_{T}+f \cdot m \cdot E$ and there are $n+f \cdot m$ shares outstanding after exercise at maturity.

We assume that there is perfect competition among the warrant holders, i.e. one particular warrant holder cannot affect the aggregate exercise volume $f \cdot m$ with his or her individual decision. This form of competition among warrant holders is also considered by Constantinides (1984) and Koziol (2006a).

In addition to the literature, we introduce imperfect information about the firm value in the sense that every warrant holder cannot observe the firm value $V_{T}$ directly but obtains a signal $v$ that might differ from the true firm value $V_{T}$. Thus, we implicitly assume that there are no reliable prices of shares or other related securities available from which a warrant holder can get a more accurate estimate of $V_{T}$ than the signal 
$v$. Moreover, we have the notion that trading of the considered assets only takes place (if at all) very rarely. Since this strong form of illiquidity might imply that any trade has a substantial opposing price impact, we suppose that (almost all) investors are therefore (effectively) prevented from selling their positions. Thus, the particular possibility to short sell a share and exercise a warrant afterwards to obtain a certain exercise value is therefore ruled out. These properties especially refer to small firms having a relatively high business risk. Since according to Standard \& Poor's, warrants are primarily issued by small and risky firms, the problem of imperfect information about the firm value is supposed be an important issue for the practical exercise of many warrants. Moreover, this approach also applies to the optimal conversion of convertible bonds. Bancel/Mittoo (2004) and Dutordoir/Van de Gucht (2008) analyze motives for firms to issue bonds with conversion rights.

For exposition purposes, we consider three groups of warrant holders: optimists, realists, and pessimists where $\frac{1}{3} m$ warrants are held by each group. The type of a warrant holder characterizes how the real firm value $V_{T}$ is related to the signal $v$ observed by this particular warrant holder. The type $\theta$ is either equal to op in the case of an optimist, re in the case of a realist or pe in the case of a pessimist. We can think of the signal $v$, which a warrant holder of type $\theta$ for a given true firm value $V_{T}$ receives, as the outcome from the function $\omega\left(V_{T}, \theta\right)$ :

$$
v=\omega\left(V_{T}, \theta\right)= \begin{cases}V_{T}+\varepsilon, & \text { for } \theta=o p \\ V_{T}, & \text { for } \theta=r e, \\ V_{T}-\varepsilon, & \text { for } \theta=p e .\end{cases}
$$

In other words, realists obtain an accurate signal $v$ for the firm value $V_{T}$, while optimists overestimate the firm value by $\varepsilon$ and pessimists underestimate the firm value by the same amount $\varepsilon .^{1}$ All warrant holders are aware of the presence of imperfect information in form of the signal generating function $\omega\left(V_{T}, \theta\right)$ and know the initial warrant holdings $\frac{1}{3} m$ of each group. However, every single warrant holder does not have knowledge of his or her individual type and the signals received by other warrant holders. The assumption that investors do not know their true type is reasonable in an environment where it is either not possible for investors to

\footnotetext{
${ }^{1}$ Apparently, there are further plausible distribution functions for the warrant holders' types. For example, we can assume a continuum of investors, where the type is uniformly distributed over the investors, and still obtain a closed-form solution for the equilibrium exercise strategy. For other elegant distribution functions, we might require numerical methods to obtain the equilibrium exercise volume. However, the general insights gained from many other distribution functions are in line with those from our setup. Therefore, we think that our assumption about the form of the distribution function of warrant holders' types is not crucial for the results in this paper.
} 
pool information about individual estimates or where investors have no incentive to convey their information to the public but hope to generate further profits from it by keeping it as private information. Hence, a warrant holder does not know where his or her estimate is located relative to the estimates of the other warrant holders. As a result of the fact that a single warrant holder does not know his or her individual type, it is equally likely for him or her to be an optimist, realist or pessimist. Hence, the probability of belonging to a given type $\theta$ is $\frac{1}{3}$ for all three feasible types. Moreover, we assume that warrant holders are risk neutral meaning that they maximize their expected wealth conditional on their individual signal $v$.

The assumption that the weights of the three types of warrant holders are known by every warrant holder must not be taken literally. If investors do not perfectly know the weights of the investors' types but have a common distribution function of these weights in mind, the exercise value (and accordingly the exercise volume) can still be determined by taking the expectation over the corresponding conditional expectation with respect to every feasible weight. Hence, from the analysis of a given weight - such as in this paper - we can draw conclusions that are supposed to hold even in the case with risk about the weights of investors' types.

\subsection{Optimal Exercise Strategy}

In order to capture the effects from imperfect information on the optimal exercise decision, we first need a benchmark model which regards the case without imperfect information. After describing the standard case without imperfect information, we refer to the case with imperfect information among warrant holders.

In the case without imperfect information, i.e. $v\left(V_{T}, \theta\right)=V_{T}$ for all $\theta$, the optimal exercise strategy is to fully exercise if $v=V_{T}$ exceeds $n \cdot E$ and not to exercise if $v=V_{T}$ is below $n \cdot E$. Hence, the optimal exercise strategy can be derived by relating the (perfect) signal $v=V_{T}$ to the critical barrier $\widehat{v}=n \cdot E$ above which a full exercise is optimal. If a warrant holder obtains a signal $v$ with

$$
v>\widehat{v}=n \cdot E
$$

a full exercise is optimal because the value of a warrant $W_{T}(v, f \cdot m)$ is positive for any arbitrary total exercise volume $f \cdot m$. Accordingly, for $v<\widehat{v}$ no exercises take place as an exercise would result in a negative warrant value $W_{T}(v, f \cdot m)$. Only in the special case that $v=\widehat{v}$ holds, every exercise decision is optimal and results in the same warrant value equal to zero. 
In the next step, we derive the equilibrium exercise decision for the case of imperfect information in order to compare it with the optimal exercise rule (2) in the standard case without imperfect information. As a consequence of the fact that investors base their decisions on conditionally expected values for their gains, we employ the concept of a Bayesian Nash equilibrium. Under imperfect information, warrant holders regard their expected wealth conditional on the individual signal $v$. Hence, a full exercise is optimal if the expectation of the value of an exercised warrant exceeds zero:

$$
\mathbb{E}\left(W_{T}(\cdot) \mid v\right)>0 .
$$

Accordingly, no exercise is optimal for

$$
\mathbb{E}\left(W_{T}(\cdot) \mid v\right)<0
$$

and an arbitrary exercise decision of an individual warrant holder is optimal for

$$
\mathbb{E}\left(W_{T}(\cdot) \mid v\right)=0
$$

These relations are a consequence of the assumption of perfect competition among warrant holders which means that a single warrant holder must consider the aggregate exercise volume as given but does not affect the aggregate exercise volume. Thus, the conditional expectation $\mathbb{E}\left(W_{T}(\cdot) \mid v\right)$ does not depend on the exercise decision of an individual warrant holder. Hence, if $\mathbb{E}\left(W_{T}(\cdot) \mid v\right)$ is zero, the expected wealth from exercising a warrant is as high as the value from no warrant exercise which implies that every exercise volume is optimal for this particular warrant holder.

The main challenge in the case with imperfect information is that every warrant holder, who receives a signal $v$, not only needs to evaluate different feasible firm values $V_{T}$ but also has to account for the aggregate exercise volume that is associated with a given true firm values. This is because not only the true firm value $V_{T}$ is relevant for the value obtained with an exercised warrant but also the total exercise volume $f \cdot m$ as representation (1) indicates. For this purpose, a warrant holder with signal $v$ can account for the fact that the true firm value $V_{T}$ must be one from the following three alternatives

$$
V_{T} \in\{v-\varepsilon, v, v+\varepsilon\}
$$

where all three alternatives are equally likely for him or her. This is a consequence of the fact that each warrant holder knows (beside his or her signal) the distribution function of imperfect information $\omega\left(V_{T}, \theta\right)$ and the weights of the warrant holders' types but has no idea about the personal type $\theta$. 
To derive the optimal exercise strategies under imperfect information, it is convenient to introduce the critical signal $\bar{v}(f)$ with $f \in(0,1)$ for which the group of warrant holders receiving a signal $v=\bar{v}(f)$ can exercise a fraction $f$ of the totally held $\frac{1}{3} m$ warrants in equilibrium. In order to ensure that a partial exercise $f \in(0,1)$ is an equilibrium strategy for a signal $v=\bar{v}(f)$, the exercise condition (3) must be satisfied. We proceed in evaluating the required expectation $\mathbb{E}\left(W_{T}(\cdot) \mid v\right)$ as follows: First, we compute $\bar{v}(f)$ assuming that the unique equilibrium strategy for signals $v \leq \bar{v}(f)-\varepsilon$ and $v \geq \bar{v}(f)+\varepsilon$ is already known. Therefore, once an investor obtains a signal $v=\bar{v}(f)$, he or she can be sure about the exercise decision of all investors receiving a different signal. Then, we show in Appendix A that these choices for the assumed exercise behavior are in fact the unique equilibrium strategies. Since for $v=\bar{v}(f)$ a partial exercise takes place, we suppose that for warrant holders with a less favorable signal $v \leq \bar{v}(f)-\varepsilon$ no exercise is optimal but a complete exercise is optimal for warrant holders with a more favorable signal equal to $v \geq \bar{v}(f)+\varepsilon$.

Consequently, the exercise condition (3) yields:

$$
\begin{aligned}
\mathbb{E}\left(W_{T}(\cdot) \mid \bar{v}(f)\right) & =\frac{1}{3} \cdot W_{T}\left(\bar{v}(f)-\varepsilon, f \cdot \frac{1}{3} m\right) \\
& +\frac{1}{3} \cdot W_{T}\left(\bar{v}(f), f \cdot \frac{1}{3} m+\frac{1}{3} m\right) \\
& +\frac{1}{3} \cdot W_{T}\left(\bar{v}(f)+\varepsilon, f \cdot \frac{1}{3} m+\frac{2}{3} m\right) \\
& =0 .
\end{aligned}
$$

The representation of the conditional expectation $\mathbb{E}\left(W_{T}(\cdot) \mid \bar{v}(f)\right)$ results from the following considerations: The warrant holder with the signal $v=\bar{v}(f)$ knows that the warrant holders of his or her type exercise $f \cdot \frac{1}{3} m$ warrants in total by the definition of the critical signal $\bar{v}(f)$. If, however, the true firm value is $\bar{v}(f)-\varepsilon$, no further warrants will be exercised. This is due to the fact that in this case the warrant holder with the signal $\bar{v}(f)$ is an optimist and the other two groups of investors obtain worse signals equal to $\bar{v}(f)-\varepsilon$ or $\bar{v}(f)-2 \varepsilon$. By assumption, no warrants are exercised for these less favorable signals. As a consequence, the resulting warrant value equals $W_{T}\left(\bar{v}(f)-\varepsilon, f \cdot \frac{1}{3} m\right)$ as the true firm value is $\bar{v}(f)-\varepsilon$ and the total exercise volume equals $f \cdot \frac{1}{3} m$. This event, that the warrant holder with a given signal $v=\bar{v}(f)$ is an optimist, has a probability equal to $\frac{1}{3}$. Moreover, it is possible that the warrant holder with the signal $v=\bar{v}(f)$ is a realist so that the warrant value is $W_{T}\left(\bar{v}(f), f \cdot \frac{1}{3} m+\frac{1}{3} m\right)$ because the realists exercise $f \cdot \frac{1}{3} m$ warrants, where the group of the optimists exercises additional $\frac{1}{3} m$ warrants. Accordingly, in the case that the warrant holder with signal $v=\bar{v}(f)$ is a pessimist, the warrant value is 
$W_{T}\left(\bar{v}(f)+\varepsilon, f \cdot \frac{1}{3} m+\frac{2}{3} m\right)$.

Again, this formula for the conditional expectation of the value of an exercised warrant indicates that not only the expected firm value $v$ is relevant for the conditional expectation but also the exercise volume that is associated with a feasible true firm value. In particular, a more favorable true firm value is associated with a higher exercise volume. This relationship that a higher true firm value corresponds to a higher total exercise volume has a negative effect on the expected value of an exercised warrant. The economic intuition behind this fundamental insight is that positive gains from an exercise, which are realized if the true firm value is relatively high, are divided among more shareholders than losses in the case of a relatively low true firm value. ${ }^{2}$ In particular, the conditionally expected value of an exercised warrant is therefore lower than the exercise value where the expected firm value and expected total exercise volume are inserted for the true firm value and true exercise volume, respectively.

Since every warrant holder with a signal $v=\bar{v}(f)$ knows that the conditional expectation of the value of an exercised warrant is as high as the value of a non-exercised warrant (namely zero), he or she can follow every arbitrary exercise strategy. In particular, a strategy where each warrant holder with a signal $v=\bar{v}(f)$ exercises a fraction $f$ of his or her warrants is optimal on the individual level. As a result, for a signal $\bar{v}(f)$, that is derived from (4), an exercise of a fraction $f$ of all warrants is an equilibrium strategy. Regarding representation (1) for the value of an exercised warrant and condition (4), we can solve for the critical signal $\bar{v}(f)$ which amounts to

$$
\bar{v}(f)=n \cdot E+\varepsilon \frac{2 m^{2}+6 n m+2 f \cdot m^{2}}{27 n^{2}+18 n m+2 m^{2}+f \cdot\left(6 m^{2}+18 n m\right)+3 f^{2} \cdot m^{2}} .
$$

The critical barrier $\bar{v}(f)$ for the exercise strategy in the case of imperfect information equals the term from the exercise strategy $\widehat{v}=n \cdot E$ in the case without imperfect information plus an additional component proportional to the degree $\varepsilon$ of imperfect information. Note that the factor $\frac{2 m^{2}+6 n m+2 f \cdot m^{2}}{27 n^{2}+18 n m+2 m^{2}+f \cdot\left(6 m^{2}+18 n m\right)+3 f^{2} \cdot m^{2}}$ by which $\bar{v}(f)$ increases in $\varepsilon$ strictly lies between zero and one for any feasible $f \in[0,1]$.

Before regarding the economic insights of the equilibrium exercise strategies, it is useful to evaluate the behavior of $\bar{v}(f)$ in $f$. One can show that the derivative $\frac{\partial \bar{v}(f)}{\partial f}$

\footnotetext{
${ }^{2}$ This positive relationship between the signal an individual warrant holder obtains and the supposed aggregate exercise volume is the main reason for the impact of imperfect information. We emphasize that this major impact does not refer to the specific model setup in this paper but it is rather a general outcome in the presence of imperfect information.
} 
is always negative

$$
\frac{\partial \bar{v}(f)}{\partial f}<0
$$

The intuition for this relation is as follows: The more warrants $f \cdot \frac{1}{3} m$ are exercised by a group with signal $\bar{v}(f)$, the less strong are the relative differences between the total number $n+\frac{1}{3} \cdot f \cdot m$ of shares in the case of a less favorable true firm value $V_{T}=\bar{v}(f)-\varepsilon$ and the number of shares $n+\frac{1}{3} \cdot f \cdot m+\frac{2}{3} \cdot m$ for a more favorable firm value $V_{T}=\bar{v}(f)+\varepsilon$. In fact, the ratio between the number of shares for a favorable and a less favorable true firm value is

$$
\frac{n+\frac{1}{3} \cdot f \cdot m+\frac{2}{3} \cdot m}{n+\frac{1}{3} \cdot f \cdot m},
$$

which decreases with a higher fraction $f$ of exercised warrants. As explained above, the expectation $\mathbb{E}\left(W_{T}(\cdot) \mid \bar{v}(f)\right)$ suffers from a higher difference between the number of shares in a favorable and in a less favorable state, because losses are less strongly shared by other investors than gains from exercise. Therefore, a lower signal $\bar{v}(f)$ is sufficient for an exercise $\left(\mathbb{E}\left(W_{T}(\cdot) \mid \bar{v}(f)\right)=0\right)$ if the exercise volume $f$ is high and the effect from the relative difference $\frac{n+\frac{1}{3} \cdot f \cdot m+\frac{2}{3} \cdot m}{n+\frac{1}{3} \cdot f \cdot m}$ is less strong. The negative sign of the derivative $\frac{\partial \bar{v}(f)}{\partial f}$ implies that

$$
\bar{v}(f) \geq \bar{v}(1)
$$

holds for all $f \in[0,1]$.

The following proposition states the major insights from imperfect information on the equilibrium exercise behavior:

Proposition 1 The equilibrium exercise behavior under imperfect information about the firm value at maturity exhibits the following properties:

(i) Warrant holders receiving a signal $v \notin(\bar{v}(1), \bar{v}(0))$ follow a unique block strategy. No exercise is optimal for signals below those from that interval and a complete exercise is optimal for signals $v$ higher than those in this interval. As a consequence, for signals $v \in(\widehat{v}, \bar{v}(1))$ a complete exercise is the optimal exercise strategy without imperfect information but no exercise is the unique equilibrium strategy with imperfect information.

(ii) There are three equilibrium strategies for warrant holders with a signal $v \in$ $(\bar{v}(1), \bar{v}(0))$ namely a complete exercise, no exercise, and a partial exercise. If a partial exercise takes place in equilibrium, the exercise volume can be lower for a more favorable signal. 
(iii) If the degree $\varepsilon$ of imperfect information increases, the length of both the range $(\widehat{v}, \bar{v}(1))$ for which the exercise behavior under imperfect information and without imperfect information differs and the range $(\bar{v}(1), \bar{v}(0))$ with multiple equilibria will be extended.

The first part of the proposition follows from the fact that for all signals $v<\bar{v}(1) \leq$ $\bar{v}(f)$, the conditional expectation $\mathbb{E}\left(W_{T}(\cdot) \mid v\right)$ of the value of an exercised warrant is negative. This is because $\mathbb{E}\left(W_{T}(\cdot) \mid v\right)$ is zero for the critical values $\bar{v}(f)$ and it apparently suffers from a less favorable signal. Therefore, the unique equilibrium strategy for warrant holders with a signal $v<\bar{v}(1)$ is not to exercise.

Conversely, for signals $v$ higher than $\bar{v}(0)$, a complete exercise $f^{*}=1$ is the unique optimal exercise strategy in equilibrium. Since in this case $v$ is above $\bar{v}(f)$ for any feasible $f$, a complete exercise is the unique equilibrium for warrant holders of the group with signal $v$. This is because the conditionally expected value of an exercised warrant is positive independent of the exercise decisions of the other warrant holders of this group.

However, according to part (ii) of the proposition a unique block exercise strategy — as it is optimal for signals $v \notin(\bar{v}(1), \bar{v}(0))$ — does not exist for signals $v \in$ $(\bar{v}(1), \bar{v}(0))$. For those signals there are three equilibrium strategies, $f^{*}=0, f^{*}=1$, and a partial exercise $f^{*} \in(0,1)$. Since $v$ out of this range is higher than $\bar{v}(1)$ an exercise is strictly better than no exercise given that all other warrant holders with the signal $v$ exercise. Hence, a complete exercise $f^{*}=1$ is a Bayesian Nash equilibrium strategy. The strategy not to exercise $f^{*}=0$ is also an equilibrium strategy because for $v<\bar{v}(0)$ the expected value of an exercised warrant is below zero given that all other warrant holders with a signal equal to $v$ do not exercise. Additionally, $f^{*}$ with

$$
v=\bar{v}\left(f^{*}\right)
$$

is a further equilibrium strategy because the expected gain from a warrant exercise equals zero if a fraction $f^{*}$ of the warrants are exercised by the group with a signal $\bar{v}\left(f^{*}\right)$.

If an equilibrium with a partial exercise $0<f^{*}<1$ occurs, then a slightly better signal $v$ can result in a lower exercise volume $f^{*}$. Formally, this follows from the fact that the critically exercise barrier $\bar{v}(f)$ declines in $f$. Hence, a more favorable signal can be associated with lower fraction $f$ of exercises.

The validity of part (iii) of the proposition can be illustrated by writing the length of the interval $(\widehat{v}, \bar{v}(1))$ for which the exercise behavior under imperfect information 
and without imperfect information differs and the length of the interval $(\bar{v}(1), \bar{v}(0))$ with multiple equilibria as follows:

$$
\begin{aligned}
\bar{v}(1)-\widehat{v} & =\varepsilon \frac{4 m^{2}+6 n m}{27 n^{2}+36 n m+11 m^{2}} \\
\bar{v}(0)-\bar{v}(1) & =\varepsilon\left(\frac{2 m^{2}+6 n m}{27 n^{2}+18 n m+2 m^{2}}-\frac{4 m^{2}+6 n m}{27 n^{2}+36 n m+11 m^{2}}\right) .
\end{aligned}
$$

These representations reveal that the length of these intervals is proportionate to the degree $\varepsilon$ of imperfect information.

In what follows, we illustrate this proposition by Figure 1 and discuss the economic implications of it.

Figure 1: Equilibrium Exercise Strategies at Maturity

The diagram shows the fraction $f^{*}$ of exercised warrants in all feasible Nash equilibria as a function of the signal $v$. The solid line refers to the case with imperfect information, while the dashed line denotes the case without imperfect information. The parameter values are: $E=100, n=100, m=100$, and $\varepsilon=1500$. The critical signals are $\widehat{v}=10000$, $\bar{v}(0)=10255.3$, and $\bar{v}(1)=10202.7$.

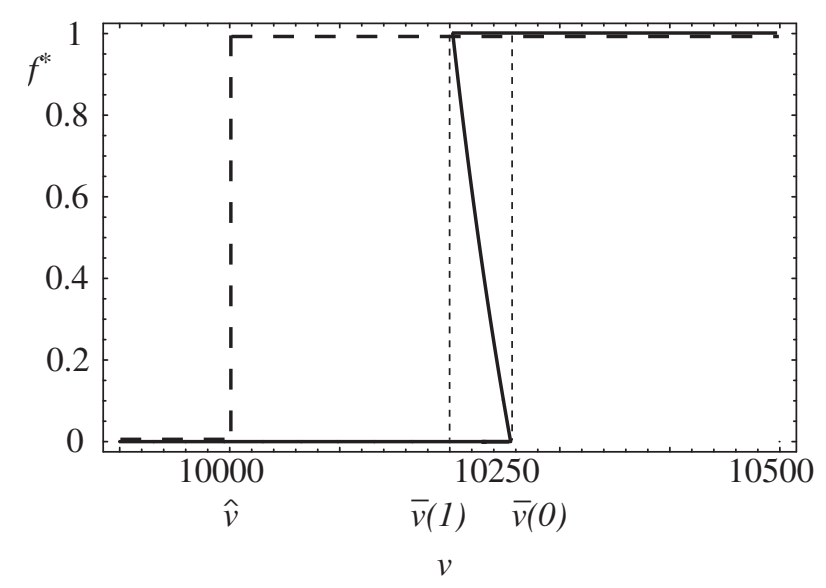

The dashed line in Figure 1 indicates the optimal exercise strategy $f^{*}$ for the case without imperfect information, i.e. for firm values $v$ below $\widehat{v}=n \cdot E$ no exercise is optimal but a complete exercise is optimal for higher firm values.

In the case with imperfect information no exercises take place for signals below $\bar{v}(1)$. Thus, under imperfect information a strictly higher signal $v \geq \bar{v}(f)$ than without imperfect information is required for a warrant exercise. This outcome, that for some signals the optimal exercise behavior differs between the cases with and without imperfect, is a first important finding from the analysis of imperfect information. The rationale for this optimal strategy is that warrant holders in the 
case of imperfect information suffer from the fact that the total exercise volume is higher if the true firm value is higher. Thus, warrant holders bear the risk that gains from an exercise must be shared with relatively many other shareholders, while losses due to a low true firm value are less strongly divided by other shareholders because of a lower exercise volume. As a result, warrant holders are aware of the fact that they exercise (on average) later relative to the case without imperfect information, i.e. there are signals $v$ with $\bar{v}(1)>v>\widehat{v}$ for which a gain from exercise is more likely than a loss from exercise. In fact the probability for a loss equals $\frac{1}{3}$ where the probability for a gain is $\frac{2}{3}$. However, the asymmetry between potential gains and losses prevents the warrant holders from an exercise as in the case without imperfect information. This result is related to the findings by Hubert/Schäfer (2002) and Morris/Shin (2004) who analyze early terminations of bank loans.

For signals $v$ higher than $\bar{v}(1)$ there is a second important range

$$
v \in(\bar{v}(1), \bar{v}(0))
$$

because for these signals three equilibrium strategies exist. The intuition for why multiple equilibria exist is as follows: For a warrant holder not only the individual exercise strategy is relevant but also the total exercise volume. As explained above, a low total exercise volume results in a higher relative difference (5) between the aggregate exercise volume for a high and a low true firm value. The consequence of a higher suchlike difference is that the important asymmetry between gains and losses from a warrant exercise for a high and a low firm value is more pronounced. Therefore, if all other warrant holders who get a signal $v \in(\bar{v}(1), \bar{v}(0))$ do not exercise, an individual warrant holder is relatively strongly exposed to the disadvantages from the asymmetry between potential gains and losses. Therefore, the conditional expectation of the value of an exercised warrant is negative. As a consequence, every individual warrant holder with this signal has no incentive to exercise his or her warrants given all other warrant holders do not exercise. Conversely, if all the other warrant holders with such a signal exercise, the asymmetry between gains and losses is less severe. Hence, the conditional expectation of the value of an exercised warrant is positive so that for all warrant holders with this signal an exercise is optimal. Finally, there is a third, 'intermediate' equilibrium strategy with a partial exercise $0<f^{*}<1$ for which the problem from the asymmetry between gains and losses is still present but less severe than in the case with no exercises.

Figure 1 shows that for signals $v \in(\bar{v}(1), \bar{v}(0))$ a complete exercise, no exercise, and a partial exercise are equilibrium strategies. Remarkably, if a partial exercise takes place, the exercise volume declines with the signal $v$. This relationship is surprising 
because typically the optimal exercise volume increases with a more favorable firm value (see e.g. Koziol (2006a)). The intuition for this outcome is that in this equilibrium the warrant holders - as explained above — suffer from a lower aggregate exercise volume $f^{*} \cdot \frac{1}{3} m$ and benefit from a more favorable signal $v$. To have a lower aggregate exercise volume $f^{*}$ in equilibrium, the signal $v$ must be higher to keep the conditionally expected warrant value at zero as it is required by the equilibrium condition in 3 . Thus, $f^{*}$ declines with $v$.

These three equilibria are associated with different conditional expectations of the value obtained with a warrant. While in the equilibrium with a complete exercise $f^{*}=1$, the warrant holders obtain a strictly positive value, the value in the case with partial exercise $f^{*} \in(0,1)$ is equal to zero, and in the case without exercise $f^{*}=0$ warrant holders also obtain a value equal to zero because the conditionally expected value of an exercised warrant were negative. As a consequence, even though strategies with a partial exercise $f^{*} \in(0,1)$ can be an equilibrium, the equilibrium strategy that results in the highest expected warrant value is a block strategy. A block strategy means that every particular warrant holder of a group with signal $v$ follows the same strategy either to fully exercise or not to exercise at all. Thus, a partial exercise volume $f \in(0,1)$ cannot occur under a block strategy.

Finally Figure 1 shows that for signals $v$ higher than $\bar{v}(0)$, a complete exercise $f^{*}=1$ is the unique optimal exercise strategy in equilibrium.

If the degree $\varepsilon$ of imperfect information rises, the effects of imperfect information are more pronounced. This property concerns both the length of the region $(\widehat{v}, \bar{v}(1))$ with signals for which the optimal exercise strategies with and without imperfect information deviate and the length of the region $(\bar{v}(1), \bar{v}(0))$ with multiple equilibrium strategies which increase with $\varepsilon$.

\subsection{Impact on Warrant Values}

The fact that the exercise behavior of every warrant holder is impacted by imperfect information, raises the question how this exercise strategy affects warrant values relative to the case without imperfect information. Intuitively, the information problem means that warrant holders might make wrong exercise decisions so that the warrant value is supposed to be lower. Having a closer look at this issue, however, it is thinkable that a given warrant holder might benefit from the suboptimal exercise behavior of the other investors. Given that this is possible, the information problem can have a positive effect on the warrant value in some states. In what follows we 
formally analyze the impact from imperfect information on the warrant value. For this purpose, we compute the expected gain that a (risk-neutral) warrant holder obtains at maturity $T$ with a warrant for a given signal $v$. Then, we relate the expected gain under imperfect information to the exercise gain when the true firm value is observable. To avoid a discussion of ambiguous exercise strategies, we assume that all warrant holders follow the most favorable strategy to fully exercise their warrant holdings if their signal $v$ exceeds $\bar{v}(1)$.

The expected gain $E G_{T}(v)$ a warrant holder obtains with this strategy is:

$$
E G_{T}(v)= \begin{cases}0, & \text { for } v \leq \bar{v}(1) \\ E G_{T}^{(1)}(v), & \text { for } \bar{v}(1)<v \leq \bar{v}(1)+\varepsilon \\ E G_{T}^{(2)}(v), & \text { for } \bar{v}(1)+\varepsilon<v \leq \bar{v}(1)+2 \cdot \varepsilon \\ E G_{T}^{(3)}(v), & \text { for } v>\bar{v}(1)+2 \cdot \varepsilon\end{cases}
$$

where

$$
\begin{aligned}
E G_{T}^{(1)}(v) & =\frac{1}{3} \cdot \frac{v-\varepsilon+\frac{1}{3} m \cdot E}{n+\frac{1}{3} m}+\frac{1}{3} \cdot \frac{v+\frac{2}{3} m \cdot E}{n+\frac{2}{3} m} \\
& +\frac{1}{3} \cdot \frac{v+\varepsilon+m \cdot E}{n+m}-E \\
E G_{T}^{(2)}(v) & =\frac{1}{3} \cdot \frac{v-\varepsilon+\frac{2}{3} m \cdot E}{n+\frac{2}{3} m}+\frac{1}{3} \cdot \frac{v+m \cdot E}{n+m} \\
& +\frac{1}{3} \cdot \frac{v+\varepsilon+m \cdot E}{n+m}-E \\
E G_{T}^{(3)}(v) & =\frac{v+m \cdot E}{n+m}-E .
\end{aligned}
$$

In the case of a signal $v$ not above the exercise barrier $\bar{v}(1)$, the warrant holder does not exercise and obtains a value equal to zero with certainty. In the other cases, the warrant holder knows that for a signal $v$, only the firm values $v-\varepsilon, v$, and $v+\varepsilon$ can be the true firm values with equal probability. In the first case for a signal $v$ with $\bar{v}(1)<v \leq \bar{v}(1)+\varepsilon$, the warrant holder knows that only her group exercises if she is an optimist because the true firm value is $v-\varepsilon$ and the warrant holders of a different type get a signal $v<\bar{v}(1)$. Therefore, the aggregate exercise volume is $\frac{1}{3} m$. If she is a realist, i.e. the true firm value is $v$, the total exercise volume is $\frac{2}{3} m$, and in the case of a true firm value equal to $v+\varepsilon$ every warrant holder exercises. We can determine $E G_{2}(v)$ in the case of $\bar{v}(1)+\varepsilon<v \leq \bar{v}(1)+2 \cdot \varepsilon$ in an analogous way. For $v>\bar{v}(1)+2 \cdot \varepsilon$, every warrant holder knows that the signal is so high that every warrant holder will exercise so that the aggregate exercise volume equals $m$.

Without imperfect information, all warrant holders exercise if the signal $v$ they obtain, which is equal to the true firm value $V_{T}$, exceeds the threshold $\widehat{v}=n \cdot E$. 
Hence, the certain exercise value $E V_{T}(v)$ a warrant holder obtains without imperfect information is

$$
E V_{T}(v)=\max \left(\frac{v+m \cdot E}{n+m}-E, 0\right) .
$$

Figure 2 shows the expected gain $E G_{T}(v)$ from an exercise in the case of imperfect information and the exercise value $E V_{T}(v)$ without imperfect information for a signal $v$. The exercise value $E V_{T}(v)$ has the well-known shape. It is zero for firm values $v$ below $\widehat{v}$ and then it is linearly increasing. The expected gain $E G_{T}(v)$ from an exercise under imperfect information is zero for signals until $v=\bar{v}(1)$. Then it linearly increases until $\bar{v}(1)+\varepsilon$. At this barrier, $E G_{T}(v)$ has a drop because some warrant holder with a less attractive signal start to exercise so that the total expected exercise gain is shared with more investors. Then $E G_{T}(v)$ linearly increases again until a signal $v=\bar{v}(1)+2 \varepsilon$. For higher signals, a complete exercise takes place so that the expected exercise gain is like in the case without imperfect information.

Figure 2: Expected Gains With and Without Imperfect Information

The diagram shows the expected gain $E G_{T}(v)$ obtained with a warrant under imperfect information (solid line) and the exercise gain $E V_{T}(v)$ in the case without imperfect information (dashed line) as a function of the signal $v$. The parameter values are: $E=100$, $n=100, m=100$, and $\varepsilon=1500$. The critical signals are $\widehat{v}=10000, \bar{v}(0)=10255.3$, and $\bar{v}(1)=10202.7$.

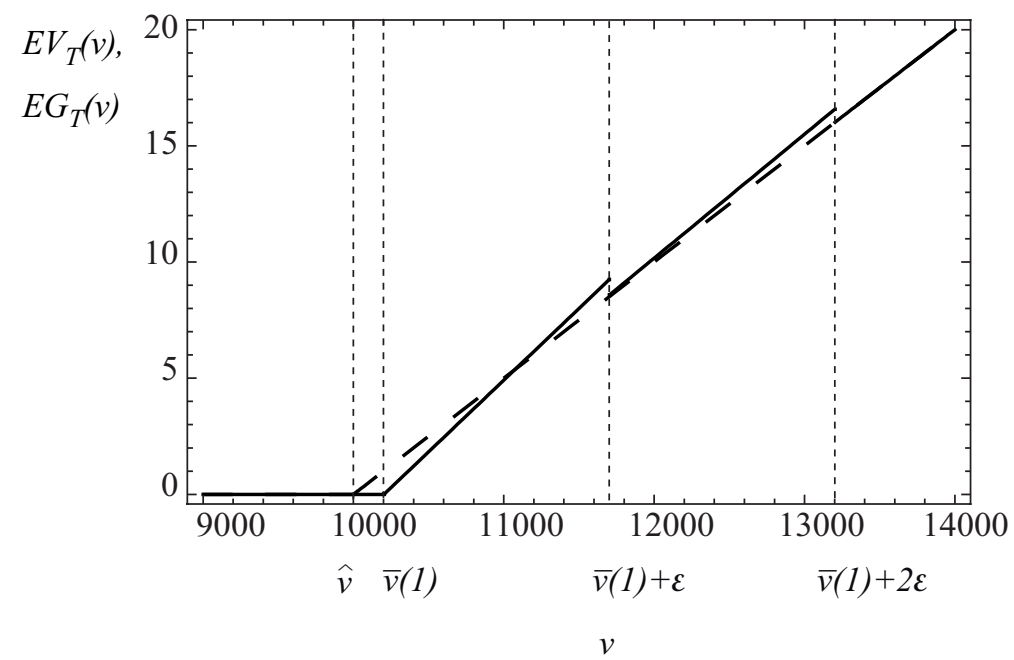

A comparison of the expected gain $E G_{T}(v)$ and the exercise value $E V_{T}(v)$ reveals that for at-the-money signals which are above the barrier $\widehat{v}$ but not too distant from $\bar{v}(1), E V_{T}(v)$ is higher than $E G_{T}(v)$. This is a result of the fact that imperfect information implies a lower expected exercise value for at-the-money warrants because the exercise decisions from the other warrant holders have a negative impact. 
Moreover, the fact that warrant holders require a more favorable signal under imperfect information to exercise their warrants confirms that for signals $v \in(\widehat{v}, \bar{v}(1))$, a positive value is only obtained in the case without imperfect information rather than with imperfect information.

However, for signals $v$ which are close to $\bar{v}(1)+\varepsilon$ and below $\bar{v}(1)+2 \cdot \varepsilon$, one can show that $E G_{T}(v)$ is strictly higher than $E V_{T}(v)$. This is a result of the fact that for those signals $v>n \cdot E+\varepsilon$, the true firm value $V_{T}$ must be above $n \cdot E$. Therefore, a warrant holder knows for sure that an exercise is optimal independent of the aggregate exercise volume. However, there is still a positive probability that some warrant holders get a signal $v$ below $\bar{v}(1)$ so that they do not exercise. Consequently, the expected gain $E G_{T}(v)$ benefits from the fact that other warrant holders make a suboptimal exercise decision. These characteristics lead to the following proposition:

Proposition 2 The existence of imperfect information affects the expected value that can be obtained with a warrant. While investors with out-of-the-money and at-the-money warrants have a lower conditionally expected warrant value, investors with (sufficiently deep) in-the-money warrants obtain a higher value due to imperfect information.

This proposition can be explained as follows: In the at-the-money region, imperfect information complicates the exercise decision and which therefore results in a lower warrant value. If warrant holders, however, get a signal which indicates that an exercise is worthwhile, they obtain a higher expected value with the warrant due to suboptimal exercise decisions of other warrant holders. For those signals, the danger of a wrong individual exercise decision is very low (or is zero for $v>n \cdot E+\varepsilon$ ) but there is a positive probability for wrong exercises by other warrant holders. Therefore, those warrant holders that exercise their warrants benefit at the cost of the other warrant holders.

\section{Imperfect Information About the Dividend}

The optimal exercise of warrants before maturity primarily depends on the size of dividend payments. ${ }^{3}$ Pricing models typically assume that an exercise in a dividend date provides the warrant holder with a share including the dividend claim. This

\footnotetext{
${ }^{3}$ See Spatt/Sterbenz (1988) and Koziol (2006a) for exceptions where exercises without dividends are possible.
} 
is not consistent with warrants traded on real markets. Koziol (2006b) points out that warrants must be exercised before the end of a business year to be converted into a share including the dividend of this particular business year. Usually, the dividend for a business year is announced and paid out several month after the end of the business year. For pricing purposes the time lag between the feasible exercise date and the dividend payment is not crucial as long as the present value of the dividend payment is commonly known. However, several month before a dividend announcement, the dividend is not commonly known so that warrant holders might have different beliefs about the dividend payment. The existence of imperfect information about the dividend might have a fundamental effect on the optimal exercise decision, as dividends are a major factor for premature exercises. In what follows, we derive the optimal exercise strategy under imperfect information about the dividend and relate it to the case without imperfect information.

\subsection{Model Framework}

In this section, we regard a point in time $t=0$ before maturity $T$. The current date $t=0$ is the latest exercise date at which an exercise not only provides the share but also the dividend. Therefore, an exercise at a point in time after $t=0$ and before $t=T$ cannot be optimal. For notational convenience, we normalize the time to maturity to $T=1$. The firm generates proceeds which are completely distributed as dividends to the shareholders at time $\tau$ between $t=0$ and $T$. The present value of the total proceeds are denoted by Div. If beside the $n$ initially outstanding shares additional $f \cdot m<m$ shares are created through warrant exercise at time $t=0$, then the present value at time $t=0$ of the dividend payment per share is ${ }^{4}$

$$
\frac{D i v}{n+f \cdot m}
$$

Since the core of this section is the imperfect information on dividend payments, we abstract from imperfect information on the firm value as introduced in the previous section. In addition, it is sufficient for our purposes to model firm value uncertainty by a simple one-period binomial tree, where the current firm value can either increase by a factor $u$ or decrease by a factor $d$.

As a result of exercises at time $t=0$, the firm value after exercise at time $t=0$ can be

\footnotetext{
${ }^{4}$ This dividend assumption is of course only one feasible assumption among many others. Alternatively, the firm could sell assets to payout additional dividends. However, the general structure, that higher dividends increase the share value, while the value of a non-exercised warrant cannot increase, is always valid.
} 
higher than the firm value $V_{0}$ before exercise. We assume that the exercise proceeds $f \cdot m \cdot E$ fully increase the current firm value from $V_{0}$ to $V_{0}+f \cdot m \cdot E$. Moreover, we assume that the firm value $V_{0}+f \cdot m \cdot E$ after exercise at time $t=0$ is so that further exercises only take place after an increase of the firm value. Strictly speaking, after an increase of the firm value from $V_{0}+f \cdot m \cdot E$ to $V_{T}=u \cdot\left(V_{0}+f \cdot m \cdot E\right)$, a complete exercise is optimal, i.e. $u \cdot\left(V_{0}+f \cdot m \cdot E\right)>\widehat{v}=n \cdot E$ is true. On the contrary, a decrease of the firm value from $V_{0}+f \cdot m \cdot E$ to $V_{T}=d \cdot\left(V_{0}+f \cdot m \cdot E\right)$ results in no further exercises at maturity, i.e. $d \cdot\left(V_{0}+f \cdot m \cdot E\right)<\widehat{v}$ holds. This assumption is to avoid a discussion of different subcases for which a different exercise behavior takes place in the two states at maturity. The economic insights gained from our model also apply to the other cases.

To price an arbitrary claim under this binomial tree structure, we can use the riskneutral probability $p$ for an increase of the firm value. The risk-neutral probability $p$ follows from the following pricing relationship:

$$
V_{0}=\frac{p \cdot u \cdot V_{0}+(1-p) \cdot d \cdot V_{0}}{1+r}
$$

where $r$ is the riskfree rate. We note that the state prices $p /(1+r)$ and $(1-p) /(1+$ $r$ ), respectively, do not necessarily follow from the true probability. Rather, the state prices can be the result of the different risk preferences from the investor. ${ }^{5}$ In this framework, the values of a non-exercised warrant $W_{0}(f \cdot m)$ and a share $S_{0}($ Div,$f \cdot m)$, depending on the total dividend Div and the aggregate premature exercise volume $f \cdot m$, are given by

$$
\begin{aligned}
W_{0}(f \cdot m) & =\frac{1}{1+r} \cdot p \cdot \frac{u \cdot\left(V_{0}+f \cdot m \cdot E\right)-(n+f \cdot m) \cdot E}{n+m}, \\
S_{0}(\text { Div }, f \cdot m) & =\frac{1}{1+r} \cdot\left(p \cdot \frac{u \cdot\left(V_{0}+f \cdot m \cdot E\right)+(m-f \cdot m) \cdot E}{n+m}\right. \\
& \left.+(1-p) \cdot \frac{d \cdot\left(V_{0}+f \cdot m \cdot E\right)}{n+f \cdot m}\right)+\frac{D i v}{n+f \cdot m} .
\end{aligned}
$$

To determine these formulae for the values of a warrant and a share, we can compute the corresponding values at maturity $T$ depending on the firm value at maturity, the number of shares outstanding before exercise at maturity, and the number of outstanding shares after exercise. If the firm value increases by factor $u$, the firm value at maturity is $u \cdot\left(V_{0}+f \cdot m \cdot E\right)$, the number of shares before exercise equals $n+f \cdot m$ due to exercises at time $t=0$, and $n+m$ shares are outstanding at maturity

\footnotetext{
${ }^{5}$ At this point we emphasize that utility functions can also be considered to evaluate the optimal exercise strategy. However, the qualitative effects derived in this paper will still remain but an optimal exercise volume in closed-form will no longer be available.
} 
after exercise. In the down state, the firm value is $d \cdot\left(V_{0}+f \cdot m \cdot E\right)$ and no further warrants are exercised at maturity.

The value of a non-exercised warrant $W_{0}(f \cdot m)$ does not depend on the dividend Div because the holders of non-exercised warrants do not receive dividend payments and the firm value at maturity is not affected by Div. The higher the exercise volume $f \cdot m$ is, the higher is the warrant value $W_{0}(f \cdot m)$, as the firm value at maturity benefits from early exercises. The stock value $S_{0}(\operatorname{Div}, f \cdot m)$ obviously benefits from a higher total dividend Div and can increase or decrease in the exercise volume $f \cdot m$.

We model imperfect information about dividends in an analogous way to imperfect information about the firm value presented in the previous section. ${ }^{6}$ Therefore, we have three groups of warrants holders, which can be understood as optimists, realists, and pessimists, where $\frac{1}{3} m$ warrants are held by each group. The type $\theta$ of a warrant holder determines the signal div about the dividend he or she receives relative to the real dividend Div. According to the signal generating function

$$
d i v=\delta(\text { Div }, \theta)= \begin{cases}\text { Div }+\varepsilon, & \text { for } \theta=o p \\ \text { Div, } & \text { for } \theta=r e \\ \text { Div }-\varepsilon, & \text { for } \theta=p e\end{cases}
$$

the signal div for optimists is higher than the true dividend Div, realists obtain an unbiased signal, and the signal for pessimists div is lower than the true dividend Div. Since an individual warrant holder does not know his or her individual type, she assigns a probability equal to $\frac{1}{3}$ to each of the three events to be an optimist, a realist, or a pessimist. Again, warrant holders base their decision on the conditional expectation of the value obtained with their warrant holdings. We note that the following examples are so that the signals div, which the warrant holders obtain, always imply a positive true dividend Div. Nevertheless, a negative Div is also reasonable. In this case, Div denotes the volume of a capital increase that has to be covered by the existing shareholders.

\section{$3.2 \quad$ Optimal Exercise Strategy}

In the first step, we determine the optimal exercise strategy in the case without imperfect information. A major difference to the exercise strategy $\widehat{v}$ at maturity is that a partial exercise with a fraction $f \in(0,1)$ of the $m$ outstanding warrants can

\footnotetext{
${ }^{6}$ In line with Section 2, we assume that there are no stock and warrant prices available that convey information about other investors' beliefs and that the warrant holders can only sell their claims at a strong discount which prevents them from doing so.
} 
be the unique optimal strategy without imperfect information. To have a partial exercise $f$ with $0<f<1$ in equilibrium, the exercise value $S_{0}(D i v, f \cdot m)-E$ must be equal to the value of a non-exercised warrant $W_{0}(f \cdot m)$ for this strategy $f$ :

$$
S_{0}(\operatorname{Div}, f \cdot m)-E=W_{0}(f \cdot m) .
$$

Otherwise for $S_{0}(\operatorname{Div}, f \cdot m)-E \neq W_{0}(f \cdot m)$, every single warrant holder would decide for the alternative with the higher value. Thus, the aggregate exercise volume would either be equal to zero or $m$ and would therefore not be consistent with a total exercise volume $f \cdot m$ with $0<f \cdot m<m$. Condition (9) is not only necessary for an equilibrium in which a fraction $f$ with $0<f<1$ of the warrants are exercised but it is also sufficient. If (9) holds, then for every particular warrant holder it is optimal to exercise e.g. a fraction $f$ of his or her warrants so that $f \cdot m$ is a Nash equilibrium strategy.

Let $\widehat{\operatorname{div}}(f)$ be the critical dividend for which a fraction $f$ is exercised. Using the closed-form representations (8) for the exercise value $S_{0}(\widehat{\operatorname{div}}(f), f \cdot m)-E$ and the value $W_{0}(f \cdot m)$ of a non-exercised warrant, the critical dividend $\widehat{\operatorname{div}}(f)$ results from (9) and amounts to:

$$
\begin{aligned}
\widehat{\operatorname{div}}(f) & =f \frac{m \cdot E \cdot((1-p) \cdot(1-d)+r)}{1+r} \\
& +\frac{n \cdot E \cdot(1-p+r)+d \cdot(1-p) \cdot V_{0}}{1+r}
\end{aligned}
$$

The critical dividend $\widehat{\operatorname{div}}(f)$ for which a fraction $f$ of the $m$ warrants are exercised in equilibrium linearly increases in $f$. It is straightforward that a higher dividend Div results in a higher incentive to exercise so that a higher fraction $f$ is exercised. For dividends Div below $\widehat{d i v}(0)$, the exercise value $S_{0}($ Div, 0$)-E$ without exercises is lower than the value of a warrant $W_{0}(0)$ so that no exercise is the equilibrium strategy. Accordingly, for high dividends Div above $\widehat{d i v}(1)$, the exercise value $S_{0}($ Div,$m)-E$ after a complete exercise exceeds the corresponding value of a non-exercised warrant $W_{0}(1)$ so that a complete exercise is an equilibrium strategy. We note that the aggregate exercise strategy characterized by $\widehat{d i v}(f)$ is the unique equilibrium strategy, as obviously other equilibrium strategies cannot exist. ${ }^{7}$

In the case of imperfect information about the dividend, every warrant holder evaluates the expectation $\mathbb{E}\left(S_{0}(\cdot)-E-W_{0}(\cdot) \mid d i v\right)$ of the exercise value minus the

\footnotetext{
${ }^{7}$ In particular, $f=1$ cannot be optimal for a dividend $D i v<\widehat{d i v}(1)$ because in this case $S_{0}(D i v, m)-E-W_{0}(m)<0$ holds. Accordingly, for any dividend Div above $\widehat{d i v}(0)$ the relation $S_{0}($ Div, 0$)-E-W_{0}(0)>0$ holds and an equilibrium with no exercises cannot be optimal.
} 
value of a non-exercised warrant conditional on the individual signal div about the dividend. In the case that this expectation is positive, a complete exercise is optimal for the warrant holder, while a negative expectation means no exercise. Only in the case of a conditional expectation equal to zero a partial exercise is optimal from the perspective of warrant holders receiving a signal equal to div.

In an analogous way as for imperfect information about the firm value at maturity, we introduce a critical signal $\overline{d i v}(f)$ for which the group of warrant holders, who receives the signal $\overline{d i v}(f)$, exercise a fraction $f$ with $0<f<1$ of their $\frac{1}{3} m$ warrants. Since for a critical signal $\overline{d i v}(f)$ a partial exercise is an equilibrium for this group, the conditional expectation must equal zero:

$$
\mathbb{E}\left(S_{0}(\cdot)-E-W_{0}(\cdot) \mid \overline{d i v}(f)\right)=0 .
$$

To show the main impact of imperfect information about the dividend on the equilibrium strategy, it is helpful to impose an assumption on the degree $\varepsilon$ of imperfect information. This assumption should ensure that warrant holders, who receive the signal $\overline{\operatorname{div}}(f)$, can be sure that all warrant holders obtaining a worse signal div $\leq \overline{\operatorname{div}}(f)-\varepsilon$ will not exercise their warrants at all. Conversely, those warrant holders with a better signal div $\geq \overline{\operatorname{div}}(f)+\varepsilon$ will fully exercise their warrants. In many examples such as for the parameter values considered in Figure 3 the assumption on $\varepsilon$ is satisfied. The important advantage from this assumption is a closed-form solution for the critical dividend $\overline{d i v}(f)$ that allows for meaningful interpretations. In the case that the assumption on $\varepsilon$ does not hold, $\overline{d i v}(f)$ can still be determined numerically by evaluating multiple conditions but the main results are still valid.

To determine the critical signal $\overline{d i v}(f)$, we can specify condition (10) using our assumption on $\varepsilon$. Therefore, we obtain

$$
\begin{aligned}
\mathbb{E}\left(S_{0}(\cdot)-E-W_{0}(\cdot) \mid \overline{\operatorname{div}}(f)\right) & =\frac{1}{3}\left(S_{0}\left(\overline{\operatorname{div}}(f)-\varepsilon, f \cdot \frac{1}{3} m\right)-E\right) \\
& -\frac{1}{3} W_{0}\left(f \cdot \frac{1}{3} m\right) \\
& +\frac{1}{3}\left(S_{0}\left(\overline{\operatorname{div}}(f), f \cdot \frac{1}{3} m+\frac{1}{3} m\right)-E\right) \\
& -\frac{1}{3} W_{0}\left(f \cdot \frac{1}{3} m+f \cdot \frac{1}{3} m\right) \\
& +\frac{1}{3}\left(S_{0}\left(\overline{\operatorname{div}}(f)+\varepsilon, f \cdot \frac{1}{3} m+\frac{2}{3} m\right)-E\right) \\
& -\frac{1}{3} W_{0}\left(f \cdot \frac{1}{3} m+\frac{2}{3} m\right) \\
& =0 .
\end{aligned}
$$


The evaluation of the conditional expectation is a result of analogous considerations as for imperfect information about the firm value presented in the previous section. If a warrant holder receives the signal $\overline{d i v}(f)$, the true dividend Div can either be $\overline{d i v}(f)-\varepsilon, \overline{d i v}(f)$ or $\overline{d i v}(f)+\varepsilon$. Each of the three possible dividends are equally likely with probability $\frac{1}{3}$. Given that the warrant holder is an optimist so that the true dividend Div is $\overline{d i v}(f)-\varepsilon$, the warrant holders of the other groups get worse signals $\overline{d i v}(f)-\varepsilon$ or $\overline{d i v}(f)-2 \varepsilon$. Hence, the only exercises are from the group of the warrant holder with a signal $\overline{d i v}(f)$ which results in a total exercise volume equal to $f \cdot \frac{1}{3} m$. Accordingly, if the true dividend Div is equal to $\overline{d i v}(f)$, the warrant holders with a worse signal $\overline{d i v}(f)-\varepsilon$ do not exercise but the warrant holders with a better signal $\overline{d i v}(f)+\varepsilon$ fully exercise so that the total exercise volume is $f \cdot \frac{1}{3} m+\frac{1}{3} m$. In the case that the warrant holder with signal $\overline{\operatorname{div}}(f)$ is a pessimist, the true dividend is $\overline{d i v}(f)+\varepsilon$ and the total exercise volume is $f \cdot \frac{1}{3} m+\frac{2}{3} m$.

Using formula (8) for the share and warrant values, condition (11) results in a closedform representation for the critical signal $\overline{d i v}(f)$ given that a partial exercise $f$ with $0<f<1$ is optimal for the group with signal $\overline{\operatorname{div}}(f)$. The critical signal $\overline{\operatorname{div}}(f)$ amounts to

$$
\begin{aligned}
\overline{\operatorname{div}}(f) & =\varepsilon \frac{2 m^{2}+6 n m+2 f \cdot m^{2}}{27 n^{2}+18 n m+2 m^{2}+f \cdot\left(6 m^{2}+18 n m\right)+3 f^{2} \cdot m^{2}}-d \cdot(1-p) \cdot V_{0} \\
& +\frac{E}{(1+r) \cdot\left(27 n^{2}+18 n m+2 m^{2}+f \cdot\left(6 m^{2}+18 n m\right)+3 f^{2} \cdot m^{2}\right)} \\
& \cdot\left(n\left(\left((6-4 d) m^{2}+(27-9 d) m n+27 n^{2}\right)(1-p)+3(m+3 n)(2 m+3 n) r\right)\right. \\
& +f^{3} m^{3}((1-d)(1-p)+r) \\
& +3 f^{2} m^{2}(((1-d) m+(3-2 d) n)(1-p)+(3 n+m) r) \\
& +f m\left(2 m^{2}((1-d)(1-p)+r)+9 n^{2}((3-d)(1-p)+3 r)\right. \\
& +(6 m n((3-2 d)(1-p)+3 r))) .
\end{aligned}
$$

The structure of $\overline{d i v}(f)$ corresponds to that for $\bar{v}(f)$ derived for the case of imperfect information about the firm value. This means $\overline{d i v}(f)$ linearly increases with the degree of imperfect information $\varepsilon$, where the slope $\frac{\partial \overline{\operatorname{div}}(f)}{\partial \varepsilon}=$ $\frac{2 m^{2}+6 n m+2 f \cdot m^{2}}{27 n^{2}+18 n m+2 m^{2}+f \cdot\left(6 m^{2}+18 n m\right)+3 f^{2} \cdot m^{2}}$ coincides with the slope $\frac{\partial \bar{v}(f)}{\partial \varepsilon}$ of $\bar{v}(f)$. The reason why $\overline{\operatorname{div}}(f)$ increases with $\varepsilon$ is again that for a given signal $\overline{\operatorname{div}}(f)$ a higher dividend $\overline{d i v}(f)+\varepsilon$ is divided by more shares than in the case of an unfavorable dividend equal to $\overline{d i v}(f)-\varepsilon$. For this reason, the conditionally expected dividend payment 
per share for a given signal $\overline{d i v}(f)$

$$
\begin{aligned}
& \frac{1}{3}\left(\frac{\overline{\operatorname{div}}(f)-\varepsilon}{n+f \cdot \frac{1}{3} m}+\frac{\overline{\operatorname{div}}(f)}{n+f \cdot \frac{1}{3} m+\frac{1}{3} m}+\frac{\overline{\operatorname{div}}(f)+\varepsilon}{n+f \cdot \frac{1}{3} m+\frac{2}{3} m}\right) \\
& =\frac{1}{3}\left(\frac{\overline{\operatorname{div}}(f)}{n+f \cdot \frac{1}{3} m}+\frac{\overline{d i v}(f)}{n+f \cdot \frac{1}{3} m+\frac{1}{3} m}+\frac{\overline{d i v}(f)}{n+f \cdot \frac{1}{3} m+\frac{2}{3} m}\right) \\
& -\varepsilon \frac{1}{3}\left(\frac{1}{n+f \cdot \frac{1}{3} m}-\frac{1}{n+f \cdot \frac{1}{3} m+\frac{2}{3} m}\right)
\end{aligned}
$$

suffers from a higher $\varepsilon$ for any given $f$. Therefore, a better signal div is required for an equilibrium with a fraction $f$ of exercises if the degree of imperfect information $\varepsilon$ is higher.

Proposition 3 The equilibrium exercise behavior under imperfect information about the dividend exhibits the following properties:

(i) Under imperfect information a more favorable dividend signal div $\geq$ $\min _{f} \overline{\operatorname{div}}(f)>\widehat{\operatorname{div}}(0)$ is required than for the case without imperfect information.

For the characterization of the equilibrium strategies the sign of the difference $\overline{\operatorname{div}}(1)-\overline{\operatorname{div}}(0)$ is crucial. For a positive difference, it holds:

(ii) The structure of the equilibrium exercise strategy under imperfect information in this case is the same as without imperfect information, i.e. a unique equilibrium strategy always exists with a continuous exercise fraction $f^{*}$ that monotonically increases in the signal div from zero for low signals div to one for high signals.

(iii) If the degree $\varepsilon$ of imperfect information increases, the length of the range $(\overline{\operatorname{div}}(0), \overline{\operatorname{div}}(1))$ with a unique partial exercise strategy in equilibria declines.

If the difference $\overline{\operatorname{div}}(1)-\overline{d i v}(0)$ is negative, we have the following:

(iv) Warrant holders receiving a signal div $\notin(\overline{\operatorname{div}}(1), \overline{\text { div }}(0))$ follow a unique block strategy. No exercise is optimal for signals below those from that interval and a complete exercise is optimal for signals div higher than those in this interval. Otherwise, there are three equilibrium strategies for warrant holders with a signal div $\in(\overline{d i v}(1), \overline{d i v}(0))$ namely a complete exercise, no exercise, and a partial exercise. Nevertheless, the block strategy to fully exercise whenever the signal div exceeds $\overline{d i v}(1)$ is the equilibrium strategy that is associated with the maximum conditionally expected wealth of the warrant holders.

In the following, we briefly show the validity of this proposition and then we discuss its economic implications and interpretations. 
Part (i) of this proposition, that in the presence of imperfect information warrant holders have no incentive to exercise for a signal div $=\widehat{\operatorname{div}}(0)$ at which warrant holders without imperfect information start to exercise, can be shown by regarding the difference $\widehat{d i v}(0)-\overline{d i v}(0)$ of the corresponding critical values. One can show that this difference is negative even for $\varepsilon=0$ and it further declines with increasing $\varepsilon$. The intuition for this is again that the expected dividend per share suffers from imperfect information $\varepsilon$ as indicated in (12).

The property formulated in part (ii) of the proposition, that the optimal fraction $f^{*}$ of exercises is unique and continuously increases in div like in the case without imperfect information, is as follows: For $\overline{\operatorname{div}}(0)<\overline{\operatorname{div}}(1), \overline{\operatorname{div}}(f)$ increases with $f$. This is a direct implication of the fact that for two different signals $d i v_{1}<d i v_{2}$, condition (11) cannot be satisfied for the same $f$. This is a consequence of the property that the stock value $S_{0}(\operatorname{Div}, f \cdot m)$ increases in Div for a given exercise volume $f \cdot m$ but $W_{0}(f \cdot m)$ is independent of Div. As a result of the monotonicity of $\overline{d i v}(f)$ in $f$, we have that the aggregate exercise volume in equilibrium is uniquely given. For signals div between $\overline{d i v}(0)$ and $\overline{d i v}(1)$, a partial exercise with a fraction $f \in(0,1)$ is the Bayesian Nash equilibrium. For lower (higher) signals div no (a complete) exercise is optimal. Obviously, other equilibrium strategies cannot exist. The third part of the proposition, i.e. $\overline{d i v}(1)-\overline{d i v}(0)$ declines with the degree $\varepsilon$, can be verified by evaluating the closed form representation for $\overline{d i v}(f)$.

Part (iv) of the proposition parallels our findings for the case with imperfect information about the firm value. Since the critical signal $\overline{d i v}(f)$ for $f=1$ is below that for $f=0$, we can use the same arguments to see that a complete exercise, no exercise, and a partial exercise are equilibrium strategies for signals div $\in(\overline{\operatorname{div}}(1), \overline{\operatorname{div}}(0))$. For a complete exercise $f=1$ in the case of a signal div $>\overline{d i v}(1)$, the conditionally expected value of an exercised warrant is higher than the conditional expectation of a non-exercised warrant in the case of a partial and no exercise. This is because the warrant value $W_{0}(\cdot)$ increases in the exercise volume. Hence, the strategy with a complete exercise for $d i v>\overline{d i v}(1)$ results in the highest conditionally expected wealth among all equilibrium strategies.

As a result, the main economic consequences from imperfect information about the dividend are as follows: First, a better signal div is required by the warrant holders to exercise at least some of their warrants compared to the case without imperfect information. Again, the conditionally expected dividend payment per share suffers from the fact that for a higher true dividend more shares are outstanding compared to lower true dividend so that gains are shared with many further investors in con- 
trast to losses. This finding is consistent with those for imperfect information about the firm value where also a more favorable signal is required to have an exercise under imperfect information. A second important outcome is that the length of the range $\overline{d i v}(1)-\overline{d i v}(0)$ for which a non-block exercise strategy is required under imperfect information is shorter than $\widehat{d i v}(1)-\widehat{d i v}(0)$ without imperfect information. This is remarkable because at first glance we might expect that imperfect information complicates the exercise strategy. However, the result from the equilibrium analysis is that simple block exercise strategies can be employed for larger intervals of signals. In particular, if the degree of imperfect information is sufficiently high, i.e. $\overline{d i v}(1)-\overline{d i v}(0)<0$, then a block strategy is an equilibrium strategy for every signal. Moreover, a block strategy is also the best strategy from the perspective of the warrant holders.

In the following, we illustrate the two feasible cases with $\overline{\operatorname{div}}(1)-\overline{\operatorname{div}}(0) \geq 0$ and $\overline{d i v}(1)-\overline{d i v}(0)<0$ and discuss the associated equilibria.

Figure 3: Equilibrium Exercise Strategies Before Maturity

The diagram shows the fraction $f^{*}$ of exercised warrants in all feasible Nash equilibria as a function of the signal div. The solid line refers to the case with imperfect information, while the dashed line denotes the case without imperfect information. The parameter values are: $E=100, V_{0}=12000, n=100, m=100, \varepsilon=2000, u=2, d=0.5, r=0.05$, and $p=11 / 30$. The critical signals are $\widehat{\operatorname{div}}(0)=2888.9, \widehat{\operatorname{div}}(1)=6381.0, \overline{\operatorname{div}}(0)=4195.2$, and $\overline{\operatorname{div}}(1)=5329.9$.

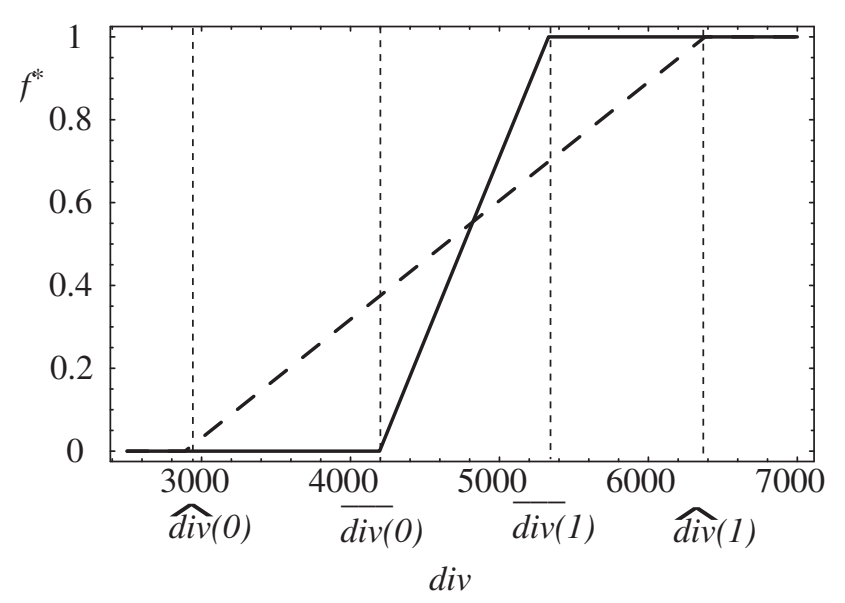

Figure 3 shows the optimal exercise decision $f^{*}$ for a given signal div with and without imperfect information. This figure refers to the case with $\overline{\operatorname{div}}(1)-\overline{\operatorname{div}}(0) \geq 0$. We note that our exercise assumption for warrant holders receiving a different signal div $\neq \overline{\operatorname{div}}(f)$ is endogenously satisfied. In particular, no exercise is optimal for warrant holders who get a signal div $\in[\overline{\operatorname{div}}(0)-\varepsilon, \overline{\operatorname{div}}(1)-\varepsilon]$ and $\operatorname{div} \in$ 
$[\overline{d i v}(0)-2 \varepsilon, \overline{d i v}(1)-2 \varepsilon]$ but a complete exercise is optimal for warrant holders with a signal $d i v \in[\overline{d i v}(0)+\varepsilon, \overline{d i v}(1)+\varepsilon]$ and $d i v \in[\overline{d i v}(0)+2 \varepsilon, \overline{d i v}(1)+2 \varepsilon]$. These properties can be verified by evaluating the sign of the conditional expectations $\mathbb{E}\left(S_{0}(\cdot)-E-W_{0}(\cdot) \mid d i v\right)$ for those dividend signals div.

We can see in this figure that the structure of the optimal exercise decision is the same with and without imperfect information as in both cases $f^{*}$ is unique and continuously increases from zero to one with the signal div. For low signals div neither with nor without imperfect information any exercises take place. Then, for signals div with

$$
d i v \in(\widehat{\operatorname{div}}(0), \overline{\operatorname{div}}(0))
$$

a partial exercise with $f>0$ is optimal without imperfect information but no exercise $f=0$ is optimal for warrant holders under imperfect information.

On the contrary, if the warrant holders get a favorable dividend signal div which results in a relatively high fraction $f$ of exercises close to one, then existence of imperfect information supports an exercise and results in a higher fraction $f$ of exercised warrants than without imperfect information, i.e. $\widehat{\operatorname{div}}(f)-\overline{\operatorname{div}}(f)>0$. One can show that $\widehat{d i v}(1)-\overline{d i v}(1)>0$ always holds for a small $\varepsilon=0$. Therefore, if the degree of imperfect information $\varepsilon$ is not very high, imperfect information enhances the exercise incentive for high $f$. This is a fundamental difference to the findings for imperfect information about the firm value where imperfect information always results in a lower exercise incentive. The intuition for the fact that imperfect information can enhance exercises is as follows: For a high signal div for which $f$ with $\operatorname{div}=\overline{\operatorname{div}}(f)$ is slightly below one, the warrant holders with this signal know that it is very likely that there are other warrant holders who receive a worse signal. As those warrant holders with a worse signal do not exercise, the aggregate exercise volume under imperfect information is lower than without imperfect information. As a consequence of the lower aggregate exercise volume, the conditionally expected dividend per share is higher and the warrant holders with signal div have a higher exercise incentive.

As a result, the main effect of imperfect information in this case is that an exercise requires a more favorable signal div than without imperfect information and a complete exercise might be optimal even for a worse signal, i.e. the signals with a partial exercise under imperfect information $(\overline{\operatorname{div}}(0), \overline{\operatorname{div}}(1))$ can be a subset of those signals $(\widehat{\operatorname{div}}(0), \widehat{\operatorname{div}}(1))$ for which a partial exercise is optimal without imperfect information.

In other cases the crucial difference $\overline{d i v}(1)-\overline{d i v}(0)$ can be negative which is the 
opposing case to figure 3. Examples for these cases are typically accompanied by relatively high degrees $\varepsilon$ of imperfect information. Since this case structurally coincides with an exercise at maturity, we do not provide an extra figure but refer to figure 1. A block exercise is an equilibrium strategy for dividend signals above $\overline{d i v}(1)$ and two more equilibrium strategies with a partial and no exercise exist for signals between $\overline{d i v}(1)$ and $\overline{d i v}(0)$. Consequently, we can see that due to the high degree of imperfect information a simple block strategy, e.g. to exercise for div $\geq \overline{d i v}(1)$, can be followed, while without imperfect information still a partial strategy must be followed.

\subsection{Impact on Warrant Values}

In line with subsection 2.3, we can compare the values of a warrant with and without imperfect information by regarding the expected values that are obtained with a warrant at time $t=0$. Technically speaking, we can follow the same steps as in section 2.3 taking into account two differences relative to an exercise at maturity: Firstly, a partial exercise can be optimal which results in the consideration of some additional subcases when determining the expected gain obtained with a warrant. Secondly, even though an exercise is not optimal, the value of a warrant is still positive due to the time value.

The outcome from this analysis is formulated in the next proposition:

Proposition 4 The existence of imperfect information affects the expected value that can be obtained with a warrant. While investors with an at-the-money warrant have a lower conditionally expected warrant value, investors with either (sufficiently deep) out-of-the-money warrants or (sufficiently deep) in-the-money warrants obtain a higher value due to imperfect information.

Since the intuition for this proposition is like that for the relationship between warrant values at maturity, we do not provide a formal proof. If warrant holders, get a signal which indicates that an exercise is optimal (or not optimal) with a relatively high probability, they obtain a higher expected value with the warrant than in the case without imperfect information. This is because there is still a severe probability for other warrant holders making wrong exercise decisions. Thus, the warrant holders benefit at the cost of warrant holders exercising their warrants suboptimally. On the contrary, for medium signals, imperfect information reduces the expected gain obtained with a warrant, because it complicates the exercise 
decision for the particular warrant holders and reduces the expected size of the dividend.

\section{Conclusion}

Investors on financial markets are often subject to imperfect information. Imperfect information can refer to the firm value if no reliable prices of shares or other appropriate instruments are available. Additionally, some month before a dividend date, investors can have different beliefs about the dividend payment which is a form of imperfect information about the dividend. Since the optimal exercise decision of corporate warrants primarily depends on the firm value and/or the dividend payment, imperfect information is a crucial factor for the optimal warrant exercise strategy in equilibrium.

The goal of this paper is to introduce imperfect information to investigate its impact on both the optimal warrant exercise strategy and the value of a warrant. For this purpose, we consider three types of warrant holders optimists, realists, and pessimists who obtain different signals about the firm value or the dividend. These investors know that imperfect information is present but they do not know their individual type. The introduction of imperfect information has fundamental consequences for the optimal strategy of warrant holders: First, a main consequence of imperfect information is that warrant holders require a more favorable signal to exercise (at least a part of) their warrants relative to the case without imperfect information. This is true for exercises at maturity with imperfect information about the firm value as well as exercises before maturity where imperfect information refers to the dividend. The reason for this property is that warrant holders account for the fact that probably some warrant holders obtain a more favorable signal and other warrant holders receive a less favorable signal. Therefore, the aggregate exercise volume is higher for a high true firm value or dividend payment but it is lower for a low true firm value or dividend payment. Hence, if the warrant holder obtains a gain from a warrant exercise the gain is shared among more warrant holders than the losses. As a result of this asymmetry, a better signal is required to compensate for this negative strategic effect.

Second, for exercises before maturity, the degree of imperfect information is crucial. Without imperfect information and if the degree of imperfect information is not too high, a unique exercise volume always exists that continuously increases in the dividend signal. However, the region of dividend signals for which a partial 
exercise is optimal under imperfect information shrinks with the degree of imperfect information. In particular, it is possible that imperfect information can enhance a complete exercise, because a group of warrant holders might want to completely exercise even for signals for which a complete exercise is not optimal without imperfect information.

Third, for a very high degree of imperfect information, the strategy, that is most favorable for the warrant holders, is a block strategy. This is remarkable because without imperfect information the unique optimal strategy is a partial exercise for some dividends.

Therefore, the two main practical implications for individual warrant holders are that due to imperfect information investors should start to exercise their warrants later than without imperfect information. As a second consequence of imperfect information we have that warrant holders can follow a common block strategy either for all dividends or at least a broader range of dividend signals than without imperfect information. Hence, imperfect information might allow warrant holders to employ a simpler strategy than without imperfect information.

Moreover, imperfect information and the resulting optimal exercise strategy impacts the value of a warrant. If the warrant is at-the-money, then imperfect information complicates the optimal exercise decision which results in a lower warrant value. However, if a warrant holder has a signal that allows for a correct exercise decision, then imperfect information increases the warrant value.

These findings can be used for further research. With a dataset of corporate warrant exercises and an appropriate proxy for the degree of imperfect information, we can test for a positive empirical relationship between a higher degree of imperfect information and a more pronounced delay of warrant exercises either before maturity or at maturity. Moreover, imperfect information is not only a relevant issue for corporate warrants but for all other corporate claims where the value of a single instrument depends on the aggregate decision of all claimholders. With a similar framework such as presented in this paper, one can also study the impact of imperfect information on those claims. 


\section{A Optimal Exercise Strategy for Signals Different From $\bar{v}(f)$}

To ensure that $\bar{v}(f)$ is in fact the critical signal, we must still show that the optimal exercise volume for signals $v \leq \bar{v}(f)-\varepsilon$ is zero and that a complete exercise is optimal for signals $v \geq \bar{v}(f)+\varepsilon$. For all signals $v \leq n \cdot E-\varepsilon(v \geq n \cdot E+\varepsilon)$ no exercise (a complete exercise) is optimal. This is due to the fact that for $v \leq n \cdot E-\varepsilon$ $(v \geq n \cdot E+\varepsilon)$ the warrant holder receiving this signal knows with certainty that the true firm value $V_{T}$ is below (above) $n \cdot E$, where according to (1) the value of an exercised warrant is negative (positive) for a true firm value $V_{T}$ below (above) $n \cdot E$ independent of the aggregate exercise volume $f \cdot m$. Since $\bar{v}(f)$ exceeds $n \cdot E$, we can conclude that for all $v \geq \bar{v}(f)+\varepsilon>n \cdot E+\varepsilon$ a complete exercise is optimal. This finding verifies the validity of our assumption that for favorable signals $v \geq \bar{v}(f)+\varepsilon$, the warrant holders fully exercise.

If an investor receives a signal equal to $v=\bar{v}(f)-\varepsilon$, there is still a positive probability equal to $\frac{1}{3}$ that the real firm value $V_{T}$ is above $n \cdot E$. For this reason, we regard the conditional expectation of the value of an exercised warrant to show that it is smaller than zero. For a given signal $\bar{v}(f)-\varepsilon$, the warrant holder knows that those warrant holders who might get an even worse signal $\bar{v}(f)-2 \varepsilon$ and potentially $\bar{v}(f)-3 \varepsilon$ will not exercise as they know for sure that $V_{T}$ is below $n \cdot E$. Accordingly, those warrant holders with a signal equal to $\bar{v}(f)+\varepsilon$ will fully exercise. Let $f_{1}$ and $f_{2}$ denote the fraction of exercised warrants of the groups who get $\bar{v}(f)-\varepsilon$ and $\bar{v}(f)$ as a signal, respectively. Then, the conditional expectation is given by

$$
\begin{aligned}
\mathbb{E}\left(W_{T}(\cdot) \mid \bar{v}(f)-\varepsilon\right) & =\frac{1}{3} \cdot W_{T}\left(\bar{v}(f)-2 \varepsilon, f_{2} \cdot \frac{1}{3} m\right) \\
& +\frac{1}{3} \cdot W_{T}\left(\bar{v}(f)-\varepsilon, f_{1} \cdot \frac{1}{3} m+f_{2} \cdot \frac{1}{3} m\right) \\
& +\frac{1}{3} \cdot W_{T}\left(\bar{v}(f), f_{1} \cdot \frac{1}{3} m+f_{2} \cdot \frac{1}{3} m+\frac{1}{3} m\right) \\
& =\frac{1}{3} \cdot \frac{\bar{v}(f)-2 \varepsilon-n \cdot E}{n+f_{2} \cdot \frac{1}{3} m}+\frac{1}{3} \cdot \frac{\bar{v}(f)-\varepsilon-n \cdot E}{n+f_{1} \cdot \frac{1}{3} m+f_{2} \cdot \frac{1}{3} m} \\
& +\frac{1}{3} \cdot \frac{\bar{v}(f)-n \cdot E}{n+f_{1} \cdot \frac{1}{3} m+f_{2} \cdot \frac{1}{3} m+\frac{1}{3} m} \quad \bar{v}(f)-n \cdot E \\
& <\frac{1}{3} \cdot \frac{\bar{v}(f)-2 \varepsilon-n \cdot E}{n+f_{2} \cdot \frac{1}{3} m}+\frac{1}{3} \cdot \frac{1}{n+f_{1} \cdot \frac{1}{3} m+f_{2} \cdot \frac{1}{3} m+\frac{1}{3} m} \\
& \leq \frac{2}{3} \cdot \frac{\bar{v}(f)-\varepsilon-n \cdot E}{n+f_{2} \cdot \frac{1}{3} m}<0 .
\end{aligned}
$$


The first inequality follows from the fact that $\frac{1}{3} \cdot \frac{\bar{v}(f)-\varepsilon-n \cdot E}{n+f_{1} \cdot \frac{1}{3} m+f_{2} \cdot \frac{1}{3} m}$ is always negative because $\bar{v}(f)-\varepsilon$ is below $n \cdot E$. The second inequality is a result of the fact that the positive term $\frac{1}{3} \cdot \frac{\bar{v}(f)-n \cdot E}{n+f_{1} \cdot \frac{1}{3} m+f_{2} \cdot \frac{1}{3} m+\frac{1}{3} m}$ is lower than $\frac{1}{3} \cdot \frac{\bar{v}(f)-n \cdot E}{n+f_{2} \cdot \frac{1}{3} m}$. The intuition for the finding that $\mathbb{E}\left(W_{T}(\cdot) \mid \bar{v}(f)-\varepsilon\right)$ is negative is as follows: If the aggregate exercise volume $f \cdot m$ were constant over all feasible signals $v$, the expected value of an exercised warrant would be $\frac{\bar{v}(f)-\varepsilon-n \cdot E}{n+f \cdot m}<0$. However, the total exercise volume monotonically increases with the signal $v$. Therefore, if the true firm value $V_{T}$ is high the aggregate exercise volume is higher than in those cases in which the true firm value $V_{T}$ is relatively low. As a consequence, gains from a positive exercise value must be shared with many other warrant holders also exercising their warrants. Conversely, in the case of a loss from an exercise, the total exercise volume is relatively low so that losses are divided among fewer investors. Since $\frac{\bar{v}(f)-\varepsilon-n \cdot E}{n+f \cdot m}$ is already negative, the effect, that more warrants are exercised if the true firm value is higher, additionally decreases the expected value of an exercised warrant.

As a result, the expected value of an exercised warrant given a signal equal to $\bar{v}(f)-\varepsilon$ is negative independent of the exercise behavior $f_{1}$ and $f_{2}$. This finding confirms our assumed exercise behavior for less favorable signals $\bar{v}(f)-\varepsilon$ to derive $\bar{v}(f)$.

\section{References}

[1] Bancel, F., Mittoo, U. R., 2004, "Why Do European Firms Issue Convertible Debt?," European Financial Management, 10, 339-373.

[2] Carlsson, H., van Damme, E., 1993, "Global Games and Equilibrium Selection," Econometrica, 61, 989-1018.

[3] Constantinides, G. M., 1984, "Warrant Exercise and Bond Conversion in Competitive Markets," Journal of Financial Economics, 13, 371-397.

[4] Cox, J. C., Rubinstein, M., 1985, Options Markets, Prentice Hall, Englewood Cliffs.

[5] Dutordoir, M., Van de Gucht, L., 2008, "Why Do Western European Firms Issue Convertibles Instead of Straight Debt or Equity?," forthcoming in European Financial Management.

[6] Emanuel, D. C., 1983, "Warrant Valuation and Exercise Strategy," Journal of Financial Economics, 12, 211-235. 
[7] Hubert, F., Schäfer, D., 2002, "Coordination Failure with Multiple-Source Lending, the Cost of Protection Against a Powerful Leader," Journal of Institutional and Theoretical Economics, 158, 256-275.

[8] Koziol, C., 2006a, "Optimal Exercise Strategies for Corporate Warrants," Quantitative Finance, 6, 37-54.

[9] Koziol, C., 2006b, "Empirical Exercise Behavior of Warrant Holders and its Consequences for Warrant Values," International Journal of Theoretical and Applied Finance, 9, 245-268.

[10] Linder, T., Trautmann, S., 2006, "Warrant Exercise and Bond Conversion in Large Trader Economies," Working Paper, University of Mainz.

[11] Morris, S., Shin, H. S. 2004, "Coordination Risk and the Price of Debt," European Economic Review, 48, 133-153.

[12] Noddings, T. C., Christoph, S. C., Noddings, J. G. 1998, The International Handbook of Convertible Securities, The Glenlake Publishing Company, Ltd.

[13] Shalem, R., 2003, "Warrant Valuation and Strategic Exercise in Continuous Time and Imperfect Competition," Working Paper, Tel Aviv University.

[14] Spatt, C. S., Sterbenz, F. P., 1988, "Warrant Exercise, Dividends, and Reinvestment Policy," Journal of Finance, 43, 493-506.

[15] Standard \& Poor's, 2007, High-Yield Bond Market Primer, McGraw-Hill Companies, Inc. 